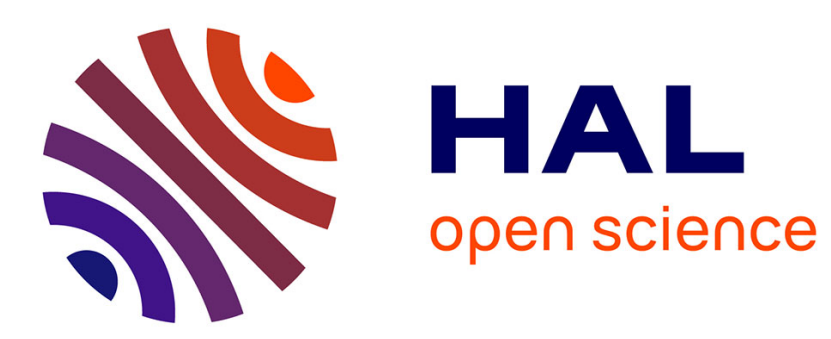

\title{
Photoluminescence properties of size-controlled silicon nanocrystals at low temperatures
}

\author{
H. Rinnert, O. Jambois, M. Vergnat
}

\section{To cite this version:}

H. Rinnert, O. Jambois, M. Vergnat. Photoluminescence properties of size-controlled silicon nanocrystals at low temperatures. Journal of Applied Physics, 2009, 106 (2), pp.023501. 10.1063/1.3169513 . hal-02164248

\section{HAL Id: hal-02164248 \\ https://hal.science/hal-02164248}

Submitted on 24 Jun 2019

HAL is a multi-disciplinary open access archive for the deposit and dissemination of scientific research documents, whether they are published or not. The documents may come from teaching and research institutions in France or abroad, or from public or private research centers.
L'archive ouverte pluridisciplinaire HAL, est destinée au dépôt et à la diffusion de documents scientifiques de niveau recherche, publiés ou non, émanant des établissements d'enseignement et de recherche français ou étrangers, des laboratoires publics ou privés. 


\title{
Photoluminescence properties of size-controlled silicon nanocrystals at low temperatures
}

\author{
H. Rinnert, ${ }^{1, a)}$ O. Jambois, ${ }^{1,2}$ and M. Vergnat ${ }^{1}$ \\ ${ }^{1}$ Institut Jean Lamour, UMR 7198, CNRS, Nancy-Université, UPV-Metz, Faculté des Sciences et Techniques, \\ Boulevard des Aiguillettes, B.P. 239, 54506 Vandouvre-lès-Nancy Cedex, France \\ ${ }^{2}$ Departament d'Electrònica, EME, IN2UB, Universitat de Barcelona, Martí i Franquès 1, \\ 08028 Barcelona, Spain
}

(Received 27 January 2009; accepted 9 June 2009; published online 16 July 2009)

This study attempts to clarify the origin of the temperature dependence of the photoluminescence (PL) spectra of silicon nanocrystals ( $\mathrm{Si}$-ncs) embedded in $\mathrm{SiO}_{2}$ from 5 to $300 \mathrm{~K}$. For this purpose, size-controlled Si-ncs with a narrow size distribution were fabricated, using the $\mathrm{SiO} / \mathrm{SiO}_{2}$ multilayer structure. The PL intensity is strongly temperature dependent and presents a maximum at around 70 $\mathrm{K}$, depending on the Si-nc size and on the excitation power. The origin of this maximum is first discussed thanks to PL dynamics study and power dependence study. The evolution of the PL energy with temperature is also discussed. In bulk semiconductors the temperature dependence of the gap is generally well represented by Varshni's law. Taking into account the quantum confinement energy, the PL energy of Si-ncs follows very well this law in the range 50-300 K. Below $50 \mathrm{~K}$, a strong discrepancy to this law is observed characterized by a strong increase in the PL energy at low temperature, which is dependent on the Si-nc size distribution. This temperature dependence of the PL energy is correlated with a decrease in the radiative rate at low temperature and is explained by a preferential saturation effect of the bigger Si-ncs. () 2009 American Institute of Physics. [DOI: $10.1063 / 1.3169513]$

\section{INTRODUCTION}

Nanostructured Si has allowed overcoming the poor optical properties of bulk $\mathrm{Si}$, by showing an enhancement of its radiative emission. This has led to a new interest in this material, to achieve the integration of silicon-based photonic devices with electronic ones on the same Si chip. The question of the origin of the light emitted by silicon nanocrystals (Si-ncs) of size lower than $5 \mathrm{~nm}$ has been involved in a great amount of investigation. It has largely been observed that the PL energy is a decreasing function of the Si-nc size, in agreement with the quantum confinement model. ${ }^{1-3}$ In the same way, it was observed that the radiative lifetime is also an increasing function of the Si-nc size, as a consequence of the $\mathbf{k}$-vector selection rule relaxation, which is more pronounced as the $\mathrm{Si}$-nc size decreases. ${ }^{2,4}$ If it is commonly accepted that the quantum confinement plays an important role in the high radiative emission yield as well as in the emission at higher energy than the Si band gap energy, a debate still exists on the origin of the PL, which could implicate surfaces states as suggested in several works. ${ }^{5-8}$ In this study, the temperature dependence of the PL characteristics is addressed. The Si-nc related PL decay time is generally strongly temperature dependent with a strong increase in the decay time at low temperature, which can be attributed to the low temperature increase in the radiative decay time. This behavior is well described by the thermal equilibrium of the lifetime of the excitonic triplet and singlet states due to the electron-hole exchange interaction, as proposed by Calcott et al. ${ }^{9}$ In this

\footnotetext{
${ }^{a)}$ Author to whom correspondence should be addressed. Electronic mail: rinnert@lpm.u-nancy.fr.
}

description, the normally forbidden triplet transition becomes slightly authorized, thanks to the spin-orbit interaction, but gives rise to a lifetime of the excited state greater than $1 \mathrm{~ms}$, whereas the singlet state is in the range of $10 \mu \mathrm{s}$. These two states are separated by some tens of meV. The upper state, the singlet one, is thermally activated so that at low temperature, only the triplet state is populated. As this state has a much longer radiative lifetime than the upper singlet state, the radiative lifetime is larger at low temperature than at high temperature. This temperature dependence of the radiative decay time has an influence on the internal quantum efficiency, defined by the ratio between the radiative decay rate value and the total decay rate value. Hence, the existence of a maximum at around $100 \mathrm{~K}$ in the temperature dependence of the PL intensity was often related in materials containing Si-ncs such as porous silicon ${ }^{10}$ or Si-ncs obtained by ion implantation of $\mathrm{Si}$ in $\mathrm{SiO}_{2} \cdot{ }^{11}$ A maximum at $150 \mathrm{~K}$ was also observed in Si-ncs obtained from laser breakdown of silane ${ }^{12}$ or by plasma enhanced chemical vapor deposition. ${ }^{13}$ The decrease in the PL intensity above $70-150 \mathrm{~K}$ is generally ascribed to a thermally activated nonradiative process. In particular, in porous silicon, it was suggested that the carriers can reach nonradiative centers such as dangling bonds, because the thermally activated transport of photogenerated carriers through the nanocrystallite network becomes important. ${ }^{14}$ However, the decrease in the PL intensity below $70 \mathrm{~K}$ is not fully understood. Some groups explained this decrease by a tunneling escape of carriers ${ }^{15,16}$ whereas Kovalev et al. ${ }^{17}$ suggested that this behavior is purely artificial and is due to a saturation of the PL, involved by the increase in 
the radiative decay time. In a first part, this paper tries to clarify the origin of the existence of such a maximum in the temperature dependence of the PL intensity.

On the other hand, the study of the evolution of the PL energy with the temperature generally gives an indication to know if the recombination is dominated by the quantum confinement, or if the recombination occurs at the interface of the Si-ncs. The temperature dependence of the semiconductor bandgap energy is well described by the phenomenological Varshni's law, ${ }^{18}$ which explains the decrease in the gap as the temperature increases. For bulk silicon, an energy gap decrease of around $60 \mathrm{meV}$ is obtained for a temperature increase from 10 to $300 \mathrm{~K}$. This energy dependence is correlated with the cumulative effect of mechanisms of electronphonon interaction and thermal lattice expansion. ${ }^{19}$ Pässler $^{20}$ precisely studied this evolution in the case of bulk silicon. In the case of Si-ncs, a few studies relate the evolution of the photoluminescence (PL) energy as a function of temperature, in particular, below $50 \mathrm{~K}$. Brongersma et al. ${ }^{11}$ observed a decrease in the PL energy equal to $60 \mathrm{meV}$ as the temperature increases from 12 to $300 \mathrm{~K}$. This evolution, mainly attributed to the decrease in the band gap energy of the Si-ncs with temperature, suggests that the PL comes from quantum confinement. Heitmann et al. ${ }^{21}$ also obtained a shift of the PL peak position from 1.472 to $1.427 \mathrm{eV}$ as the temperature increases from 4.5 to $300 \mathrm{~K}$. Moreover, they observed a significant deviation to the evolution reported by Brongersma et al. ${ }^{11}$ below $50 \mathrm{~K}$. This deviation is attributed to an additional mechanism taking into account the migration of the excitons from Si-ncs to Si-ncs, but the existence of such a mechanism in this kind of material is still a subject of controversy. ${ }^{22-24}$

In this study, we focus our attention on the temperature dependence of the PL properties of Si-ncs embedded in $\mathrm{SiO}_{2}$, in the range 5-300 K. The Si-ncs are fabricated from $\mathrm{SiO} / \mathrm{SiO}_{2}$ multilayers, which is an approach that allows the control of the Si-nc size and the obtention of a narrow size distribution, as previously shown by Zacharias et al. $^{3}$ The evolution of the PL intensity as a function of the temperature shows a dependence on the Si-nc size and on the excitation power. Moreover it is shown that the temperature dependence of the energy follows Varshni's law from 40 to $300 \mathrm{~K}$, i.e., the behavior is similar to that of bulk $\mathrm{Si}$, with an energy shift due to the quantum confinement. Below $40 \mathrm{~K}$, a clear discrepancy to Varshni's law is observed, characterized by a strong increase in the PL energy by decreasing the temperature. This behavior is dependent on the size distribution, with a more pronounced energy increase for the large size distribution. The temperature dependence of the PL energy below $40 \mathrm{~K}$ is attributed to a size distribution effect and to a preferential saturation effect of the bigger Si-ncs.

\section{EXPERIMENT}

$\mathrm{SiO} / \mathrm{SiO}_{2}$ multilayers were prepared by successive thermal evaporation of $\mathrm{SiO}$ powder and evaporation of fused silica glass performed by an electron-beam gun. The deposition rate is controlled by a quartz microbalance system and is equal to $0.1 \mathrm{~nm} \mathrm{~s}^{-1}$. The thickness of the active layer was varied from 2 to $5 \mathrm{~nm}$, whereas the $\mathrm{SiO}_{2}$ barrier thickness
TABLE I. Values of the SiO layer thickness, the Si-nc mean size, and the size dispersion for the different studied samples.

\begin{tabular}{lccc}
\hline \hline Sample & $\begin{array}{c}\text { SiO thickness } \\
(\mathrm{nm})\end{array}$ & $\begin{array}{c}\text { Mean size } \\
(\mathrm{nm})\end{array}$ & $\begin{array}{c}\text { FWHM } \\
(\mathrm{nm})\end{array}$ \\
\hline S2 & 2 & 1.8 & 1.2 \\
S3 & 3 & 3.2 & 1.3 \\
S4 & 4 & 4.1 & 1.7 \\
S5 & 5 & 4 & 4.6 \\
\hline \hline
\end{tabular}

was maintained constant and equal to $5 \mathrm{~nm}$. The silicon substrates were maintained at $100{ }^{\circ} \mathrm{C}$. For each sample, the total film thickness was around $200 \mathrm{~nm}$. The samples were then annealed at $1050{ }^{\circ} \mathrm{C}$ during 5 min under vacuum with a pressure equal to $10^{-6}$ Torr. Both continuous-wave $(\mathrm{CW})$ and time-resolved (TR) PL experiments were performed. For the $\mathrm{cw}$ experiments, the excitation was obtained with a 30 $\mathrm{mW} \mathrm{He}-\mathrm{Cd}$ laser using the $325 \mathrm{~nm}$ line. The samples were inserted in a cryostat equipped with fused silica windows with $92 \%$ transmission at the laser wavelength. Given the excitation area, the laser power reaching the sample gives rise to a photon flux equal to $1.8 \times 10^{18} \mathrm{~s}^{-1} \mathrm{~cm}^{-2}$. The excitation power can be varied using neutral density filters. For the TR-PL experiments, the sample was pumped by the 355 $\mathrm{nm}$ line of a frequency-tripled yttrium aluminum garnet (YAG):Nd laser. The laser pulse frequency, energy, and duration were typically equal to $10 \mathrm{~Hz}, 50 \mu \mathrm{J}$, and $20 \mathrm{~ns}$, respectively. The PL signal was analyzed by a monochromator equipped with a 600 grooves $/ \mathrm{mm}$ grating and by a photomultiplier tube cooled at $190 \mathrm{~K}$. The rise time of the detector is equal to around $10 \mathrm{~ns}$. The response of the detection system was precisely calibrated with a tungsten wire calibration source. The cryostat is equipped with a He flux and the temperature is controlled with an accuracy of $0.2 \mathrm{~K}$. For the experiments, the cryostat was first cooled down to $5 \mathrm{~K}$ then heated step by step up to $300 \mathrm{~K}$.

The anneal of the film at $1050{ }^{\circ} \mathrm{C}$ leads to the formation of three dimensionally confined Si-ncs, coming from the dissociation of $\mathrm{SiO}$ into $\mathrm{Si}$ and $\mathrm{SiO}_{2}{ }^{25}$ Due to the existence of the stable $\mathrm{SiO}_{2}$ barrier, their size is limited and then controlled by the SiO layer thickness. ${ }^{26}$ The transmission electron microscopy study of our samples has been presented in detail in a previous work. ${ }^{27}$ The size distribution is narrow for the sample with Si-nc size up to $4 \mathrm{~nm}$. The Si-ncs' mean diameter and the full width at half maximum of the size distribution are reported in Table I. It is important to note that, despite the control of the mean crystallite size, a size dispersion of the Si-ncs exists. This size dispersion is an increasing function of the $\mathrm{SiO}$ thickness. In particular, a full width at half maximum of the size distribution equal to 4.6 $\mathrm{nm}$ is obtained for a $\mathrm{SiO}$ thickness equal to $5 \mathrm{~nm}$. Beyond this $\mathrm{SiO}$ thickness, the Si-ncs' size is not controlled anymore and the size distribution becomes multimodal.

\section{RESULTS AND DISCUSSION}

\section{A. Temperature dependence of the decay time}

As previously shown, a blueshift of the PL energy is clearly obtained from 1.45 to $1.67 \mathrm{eV}$, as the size is reduced 

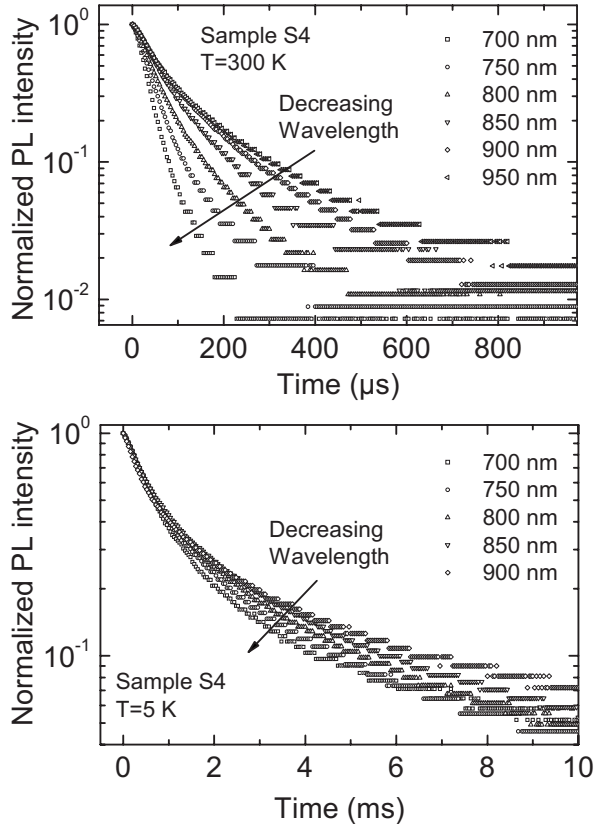

FIG. 1. PL decay profiles for sample S4 for different wavelengths, measured at 300 and $5 \mathrm{~K}$

from 4 to $2 \mathrm{~nm}$, showing that a confinement effect occurs in the samples. ${ }^{27}$ The PL energy is very similar for the $\mathrm{SiO}$ layer thicknesses equal to 4 and $5 \mathrm{~nm}$. The PL transient was first studied as a function of the wavelengths. Figures 1(a) and 1(b) show this dependence for sample S4 at room temperature and at $5 \mathrm{~K}$, respectively. The shape of the PL transient is clearly multiexponential, which is a well-known characteristic of Si-ncs. ${ }^{28-30}$ In agreement with the quantum confinement model, the decay time is a decreasing function of the wavelength both at room temperature and at $5 \mathrm{~K}$. Indeed, for the low wavelength, the PL signal is preferentially due to the small Si-ncs, which present the lower decay time, while at a larger wavelength the larger Si-ncs are measured. The dependence of the decay time on the wavelength is then mostly a consequence of the size distribution. At room temperature and at $5 \mathrm{~K}$, the characteristic decay times are in the range $10 \mu \mathrm{s}$ and $1 \mathrm{~ms}$, respectively.

The temperature dependence of the PL decay time, measured at the maximum of the PL intensity, is shown in Fig. 2, for the different Si-nc sizes. The TR-PL decay was fitted by a stretched exponential decay and only the characteristic time $\tau$ is presented here. As already observed in literature,

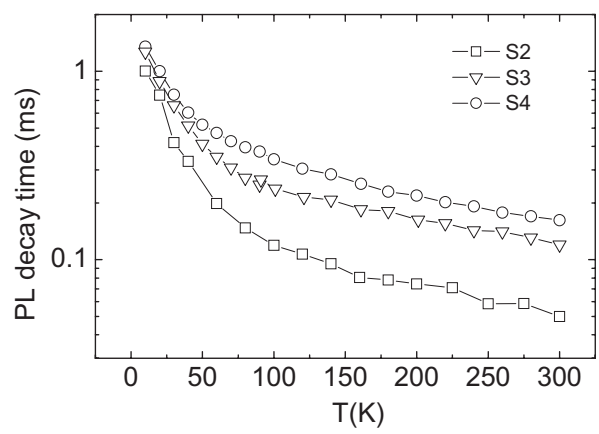

FIG. 2. Temperature dependence of the characteristic PL decay time for samples S2, S3, and S4, measured at 750,800 , and $850 \mathrm{~nm}$, respectively. the decay time is a decreasing function of the temperature with a more pronounced decrease from 5 to $50 \mathrm{~K}$. If nonradiative processes are more efficient than the radiative ones, the measured decay time is lower than the radiative decay time. In this case the PL intensity is governed by the nonradiative channels. The decrease in the decay time with temperature involves the decrease in the PL intensity, as it is the case in the temperature range between around $100 \mathrm{~K}$ and room temperature, where both PL intensity and decay time are decreasing functions of the temperature (Figs. 2 and 4). However, below $100 \mathrm{~K}$, the increase in the decay time cannot be attributed to an increase in the nonradiative decay time, because if that were the case, the PL intensity would increase monotonically by decreasing the temperature. As this is not observed, this suggests that the increase in the decay time below $100 \mathrm{~K}$ is due to an increase in the radiative lifetime. Thus, we conclude that at low temperature, the dependence of the decay time is controlled by the radiative processes. Qualitatively, the model proposed by Calcott et al., ${ }^{9}$ describing the temperature dependence of the radiative decay time, satisfactorily explains the temperature dependence of the decay time. However, the calculation of the values of the radiative decay time of the triplet and singlet states is difficult because the experimentally measured decay time also takes into account the nonradiative recombination channels, which as said before appear more likely for the high temperatures.

\section{B. Temperature dependence of the PL intensity}

CW PL experiments were performed on each sample from 5 to $300 \mathrm{~K}$ with a particularly weak temperature step to obtain a high precision on the evolution of the PL properties. Figure 3 shows the PL spectra for sample S4 for different temperatures, to present a typical evolution of the spectra with temperature, and with an excitation flux equal to 1.8 $\times 10^{18} \mathrm{~s}^{-1} \mathrm{~cm}^{-2}$. This evolution is very similar for the other $\mathrm{Si}$-nc sizes. In the next, the integrated intensity and the energy of the maximum of the PL peak will be presented as a function of the temperature. The spectra were first measured using a filter with $10 \%$ transmission of the He-Cd laser excitation, leading to a photon flux equal to 1.8 $\times 10^{17} \mathrm{~s}^{-1} \mathrm{~cm}^{-2}$. The influence of the excitation power will then be discussed.

The temperature dependence of the PL intensity is shown in Fig. 4 for the different Si-nc sizes, and for an excitation flux equal to $1.8 \times 10^{17} \mathrm{~s}^{-1} \mathrm{~cm}^{-2}$. For all the Si-nc sizes, the temperature dependence of the PL intensity presents a maximum that appears at around $70 \mathrm{~K}$. This maximum appears at a slightly lower temperature for sample S2. The decrease in the PL intensity at high temperature is generally explained by a temperature-activated nonradiative process. As said before, at low temperature, the decrease in the PL intensity needs to be discussed taking into account the radiative decay time. Assuming the $\mathrm{Si}$-ncs can be represented by a quasi-two-level system, the following rate equation can be written: 


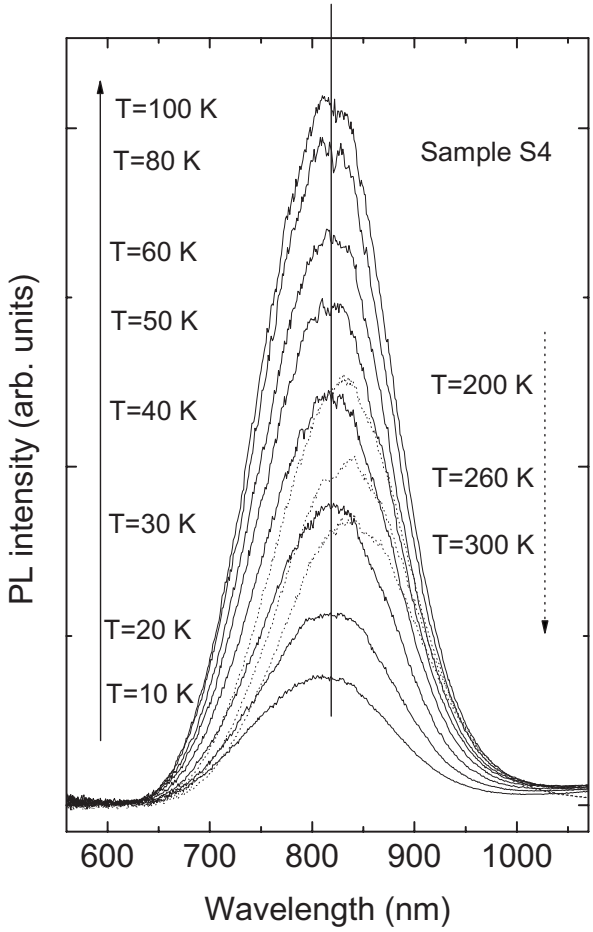

FIG. 3. Temperature dependence of the PL spectra for sample S4. The excitation photon flux is equal to $1.8 \times 10^{18} \mathrm{~cm}^{-2} \mathrm{~s}^{-1}$. For the clarity of the figure, the plain (dotted) lines correspond to the temperature from 10 to 100 $\mathrm{K}$ (from 200 to $300 \mathrm{~K}$ ).

$$
\frac{d N^{*}}{d t}=\sigma \varphi\left(N-N^{*}\right)-\frac{N^{*}}{\tau},
$$

where $N, N^{*}, \sigma, \tau$, and $\varphi$ are the total number of the Si-ncs, the number of the Si-ncs in the excited state, the Si-nc ab-
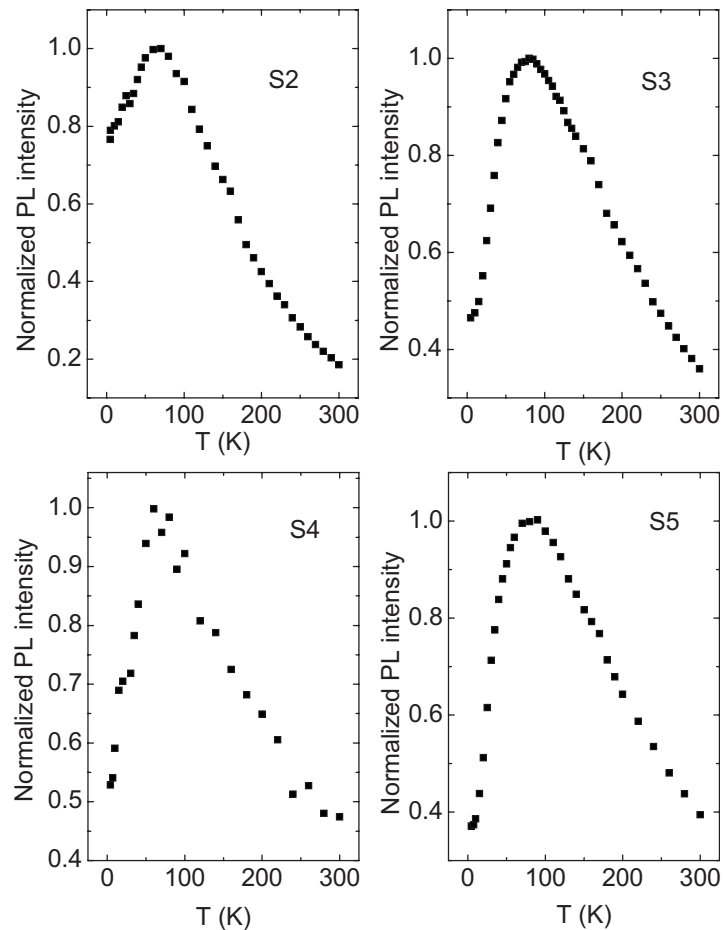

FIG. 4. Temperature dependence of the PL intensity for the different samples. The excitation photon flux is equal to $1.8 \times 10^{17} \mathrm{~cm}^{-2} \mathrm{~s}^{-1}$.

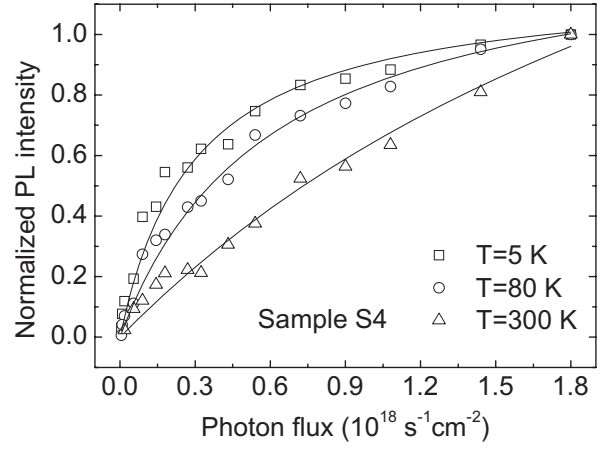

FIG. 5. Influence of the excitation power on the PL intensity for different temperatures, for sample S4. The data are normalized with respect to the PL intensity measured at the maximum of the laser power used. The lines represent the fit of the data according to expression (3) of the text.

sorption cross section at $325 \mathrm{~nm}$, the lifetime of the Si-nc excited state, and the excitation photon flux, respectively. If the PL intensity is defined by

$$
I_{\mathrm{PL}} \cong \frac{N^{*}}{\tau_{\mathrm{rad}}}
$$

where $\tau_{\text {rad }}$ is the Si-nc radiative lifetime, solving Eq. (1) for steady state conditions involves the following expression of the PL intensity:

$$
I_{\mathrm{PL}} \cong \frac{1}{\tau_{\mathrm{rad}}} \frac{\sigma \varphi \tau}{1+\sigma \varphi \tau} N
$$

For low power conditions, i.e., for

$$
\varphi \ll 1 / \sigma \tau
$$

$I_{\mathrm{PL}}$ can be approximated by

$$
I_{\mathrm{PL}} \cong \frac{1}{\tau_{\mathrm{rad}}} \sigma \varphi \tau N
$$

For a constant flux, the PL intensity is proportional to the decay time. This equation shows that for the hightemperature range (beyond $70 \mathrm{~K}$ ) where the PL intensity is a decreasing function of the temperature, the decrease in the PL intensity is well explained by the decrease in the decay time, in agreement with the fact that the nonradiative processes play an important role in the PL efficiency in this temperature range. However, at low temperature, if we assume that the decay time is mainly governed by the radiative process, $\tau \cong \tau_{\text {rad }}$, and, therefore, the PL intensity should become nearly independent of the decay time and of the temperature, as shown by Eq. (5). Hence Eq. (5) cannot explain the decrease in the PL intensity at low temperatures. Consequently, studying the influence of the excitation power is needed because expression (5) is the consequence of approximation (4), which is likely to become wrong at low temperature due to the PL decay time increase by at least one order of magnitude at low temperature.

The influence of the laser power on the PL intensity was investigated for different temperatures. The PL intensity of the maximum is presented in Fig. 5 as a function of the laser power for 5,80 , and $300 \mathrm{~K}$, for sample $\mathrm{S} 4$. The data are normalized at $100 \%$ of the laser power. It appears that, at 


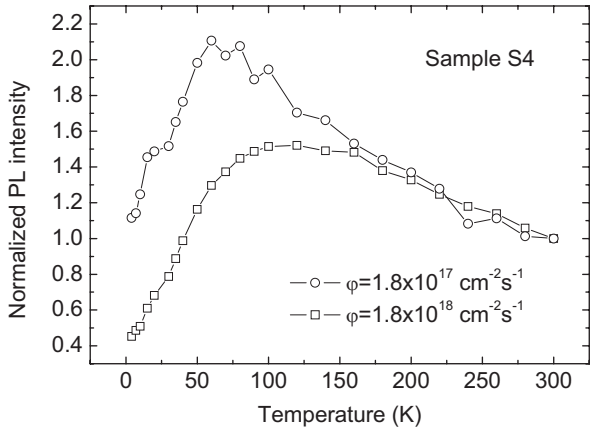

FIG. 6. Temperature dependence of the PL intensity for two different excitation photon fluxes, for sample S4.

room temperature, the PL intensity increases almost linearly with the photon flux. When the temperature is lowered at 80 $\mathrm{K}$, this evolution is no more linear. This nonlinear behavior is even more pronounced at $5 \mathrm{~K}$. The power dependence shows that for the low-temperature measurement, approximation (4) becomes wrong. Hence expression (3) was used to fit the experimental data. As shown in Fig. 5 by the continuous lines, this expression satisfactorily fits the data, allowing us to extract the product $\sigma \tau$. It is found that the $\mathrm{Si}$-nc cross section, measured at $325 \mathrm{~nm}$, is equal to $3 \times 10^{-15} \mathrm{~cm}^{2}$. For $T=5 \mathrm{~K}$, the inverse of the product $\sigma \tau$ is equal to 3 $\times 10^{17} \mathrm{~cm}^{-2} \mathrm{~s}^{-1}$, which is of the same order of magnitude than the photon flux. This clearly shows that approximation (4) is not valid at low temperature for the range of excitation power used in this study, even for the experiments performed with $10 \%$ of the laser power. At room temperature, $1 / \sigma \tau$ is equal to $3 \times 10^{18} \mathrm{~cm}^{-2} \mathrm{~s}^{-1}$ and for the experiments performed with $10 \%$ of the laser power $(\varphi=1.8$ $\times 10^{17} \mathrm{~cm}^{-2} \mathrm{~s}^{-1}$ ), the low power approximation can be used. These results concerning the power dependence of the PL intensity implicates that the temperature dependence of the PL intensity must be explained taking into account the increasing value of the PL decay time at low temperatures.

As $1 / \sigma \tau$ and $\varphi$ become very close values at low temperature, it is interesting to analyze the effect of the increase in the radiative decay time at low temperature with respect to the excitation power. Figure 6 shows the temperature dependence of the PL intensity for two different excitation powers, $10 \%$ and $100 \%$ of the laser power. The data have been normalized with respect to the value of the intensity at $300 \mathrm{~K}$. As the power dependence at $300 \mathrm{~K}$ is linear, a factor of 10 exists between both sets of data. It can be observed that from 300 to around $150 \mathrm{~K}$, the temperature dependence of the PL intensity follows a similar evolution. But it can be noticed that a strong shift of the PL intensity maximum toward the high temperature occurs when the power is increased by one order of magnitude. For the excitation power equal to $10 \%$ and $100 \%$ of the maximum power, the temperature of the maximum is equal to 70 and $110 \mathrm{~K}$, respectively. Moreover, a strong quenching of the PL occurs at low temperature for the measurement performed at high power. In this case, the $\mathrm{PL}$ at $5 \mathrm{~K}$ is less intense than the PL at $300 \mathrm{~K}$, while it is not the case for the low power measurements. It can be noticed that experiments were also performed with an excitation power equal to $1 \%$ of the maximum power. No decrease in the PL intensity at low temperature is expected. Unfortunately, the PL intensity was too low to be measured. Such a power dependence was also reported by Kovalev et al. ${ }^{17}$ in porous silicon and was attributed to the long luminescence lifetimes at low temperatures, which induce a saturation of the PL. The results presented in Fig. 4 are interpreted by the strong increase in the radiative decay time that appears at low temperature, which implicates the use of Eq. (3). This increase shows more visible effects at high power because the product $\sigma \varphi \tau$ becomes close to 1 at higher temperature for the high excitation power. In fact, the increase in the radiative lifetime at low temperature involves a decrease in the radiative recombination rate and therefore in a saturation of the PL intensity, which appears at higher temperature for the high excitation power.

Let us discuss on the Si-nc size influence on the PL intensity behavior with respect to the temperature. As shown in Fig. 4, sample S2 presents a decrease in the PL intensity appearing at lower temperature than for samples S3 and S4. Moreover this decrease is less pronounced. If we refer to the PL intensity at $300 \mathrm{~K}$, the decrease in the PL intensity at $5 \mathrm{~K}$ is more important for the larger Si-nc sizes. These results are in agreement with the major role played by the radiative decay time, which is an increasing function of the size. For example, the decay time is equal to $350 \mu$ s at $100 \mathrm{~K}$ for sample S4. The same decay time is obtained at lower temperature, i.e., $40 \mathrm{~K}$ for sample S2. Thus, the saturation effect appears at lower temperatures for sample S2, leading to a less pronounced decrease in PL intensity.

\section{Temperature dependence of the PL energy}

The temperature dependence of the energy $E_{\mathrm{PL}}(T)$ of the PL peak maximum is presented in Fig. 7 for the different Si-nc sizes. The PL energy is a decreasing function of the temperature with a stronger evolution for temperatures below $40 \mathrm{~K}$. In bulk semiconductors, the evolution of the gap with temperature is generally well described by the phenomenological Varshni's law

$$
E_{g}^{\text {bulk }}(T)=E_{g}^{\text {bulk }}(0)-\frac{\alpha T^{2}}{T+\beta},
$$

where $\alpha$ and $\beta$ are characteristic parameters. ${ }^{18}$ This law was modified by Vinã et $a l^{31}$ and by Pässler ${ }^{19}$ who obtained the following law:

$$
E_{g}^{\text {bulk }}(T)=E_{g}^{\text {bulk }}(0)-\frac{\alpha \Theta}{2}\left[\sqrt[p]{1+\left(\frac{2 T}{\Theta}\right)^{p}}-1\right],
$$

where $\alpha, p$, and $\Theta$ are empirical model parameters. The advantage of this phenomenological law is that the quasiquadratic low-temperature asymptote and the linear hightemperature asymptote of the evolution of the $\mathrm{Si}$ gap with temperature are well described. The best values that fit the experimental data on bulk silicon are $E_{g}$ bulk $(0)=1.17 \mathrm{eV}, \alpha$ $=0.3176 \mathrm{meV} / \mathrm{K}, p=2.33$, and $\Theta=405.6 \mathrm{~K}$. Taking into account the quantum confinement energy $E_{g}{ }^{\operatorname{conf}}(T)$, the law becomes 

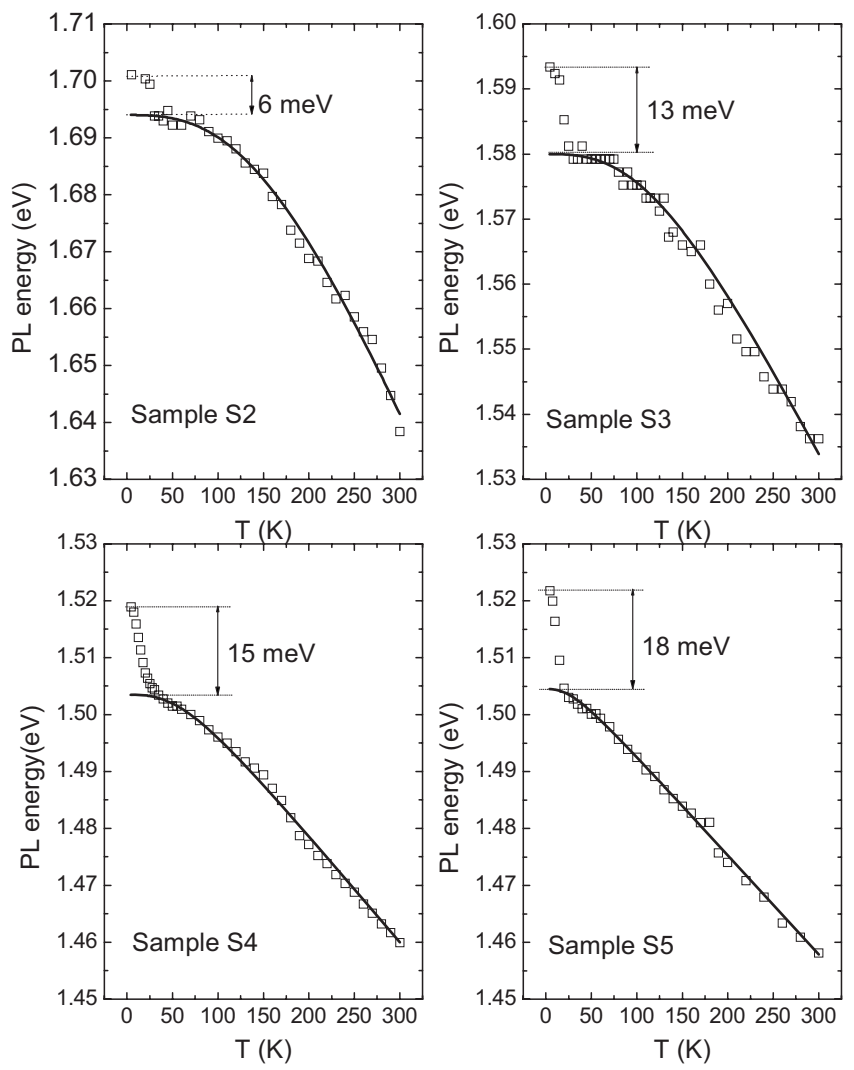

FIG. 7. Temperature dependence of the PL energy for the different samples. The curve fit is obtained according to Pässler's law (Ref. 19). The parameters of the fit are given in Table II. The energy shift between the value given by Pässler's law (Ref. 19) and the Si-nc PL energy at $5 \mathrm{~K}$ is shown for each sample.

$$
E_{g}{ }^{\text {Si-ncs }}(T)=E_{g}{ }^{\text {bulk }}(T)+E_{g}{ }^{\operatorname{conf}}(T) .
$$

For the evolution with temperature of the $E_{g}{ }^{\text {conf }}(T)$ value, we can show that a constant value can be used. Indeed, with a characteristic size of the Si-ncs equal to $L$, and assuming infinite barrier heights, the confinement energy is proportional to $L^{-2}$ and the shift of the confinement energy due to the thermal expansion of the nc can be overestimated by

$$
\left|\Delta E_{g}{ }^{\operatorname{conf}}\right| \leq 2 E_{g}{ }^{\operatorname{conf}}(T=0 \mathrm{~K}) \frac{\Delta L}{L},
$$

where $\Delta L$ is the size variation between 300 and $4 \mathrm{~K}$. Assuming a constant thermal expansion coefficient of silicon with temperature equal to $2 \times 10^{6} \mathrm{~K}^{-1}$, we obtain $\Delta L / L=6$ $\times 10^{-4}$. With a confinement energy equal to $240 \mathrm{meV}$, which is the value given for a nc with a diameter equal to $3 \mathrm{~nm},{ }^{21}$ the overestimated confinement energy shift is equal to 0.28 meV.

Due to this very low value, below the detection sensitivity, our data were fitted by the following expression:

$$
E_{\mathrm{PL}}(T)=E_{g}^{\text {conf }}+E_{g}^{\text {bulk }}(0)-\frac{\alpha \Theta}{2}\left[\sqrt[p]{1+\left(\frac{2 T}{\Theta}\right)^{p}}-1\right]
$$

The fits of our experimental results with this law are shown in Fig. 7 and the parameters of the fits are given in Table II. The values $E_{g}{ }^{\text {conf }}$ correspond to the difference between the PL energy value and the gap of bulk silicon at $300 \mathrm{~K}$. The
TABLE II. Fitting parameters of the temperature dependence of the PL energy in the $50-300 \mathrm{~K}$ range, for the different Si-nc sizes.

\begin{tabular}{lccccc}
\hline \hline Sample & $\begin{array}{c}E_{\text {confinement }}(\mathrm{eV}) \\
\text { Bulk Si }\end{array}$ & $\begin{array}{c}E_{g}(0) \\
(\mathrm{eV})\end{array}$ & $\begin{array}{c}\alpha \\
\left(\mathrm{meV} \mathrm{K}^{-1}\right)\end{array}$ & $\Theta / 2$ & $p$ \\
S2 & 0 & 1.17 & 0.317 & 202.8 & 2.33 \\
S3 & 0.524 & 1.17 & 0.4 & 200 & 2.8 \\
S4 & 0.410 & 1.17 & 0.28 & 150 & 2.8 \\
S5 & 0.333 & 1.17 & 0.19 & 73 & 2.8 \\
\hline \hline
\end{tabular}

dependence of the PL energy on temperature is very well described by Pässler's law ${ }^{19}$ in the temperature range 40-300 $\mathrm{K}$. Moreover the confinement energies are in good agreement with the already published results. ${ }^{21,32}$ Note that the sample that gives closer parameters to bulk Si for the Pässler law ${ }^{19}$ is sample S2, which has the narrower size distribution. The origin of the evolution of these parameters for the other samples is still not clear but could be assigned to the larger size distribution of these samples. However, a strong discrepancy of the temperature dependence of the PL energy on Pässler's law ${ }^{19}$ appears below $40 \mathrm{~K}$. That law predicts that the gap energy remains almost constant and equal to the gap value at $4 \mathrm{~K}$. However, we obtain a strong increase in the PL energy below $40 \mathrm{~K}$. Such an evolution has already been related by two authors. In the work of Brongersma et al., ${ }^{11}$ the PL energy decreases with temperature in the measured range $12-300 \mathrm{~K}$. A stronger decrease from 12 to around $40 \mathrm{~K}$ seems to be visible, but is not commented. Heitmann et al. ${ }^{21}$ observed a clear dependence of the PL energy on temperature. In the range $40-300 \mathrm{~K}$, their values were well fitted by the type expression of Viña et al. ${ }^{31}$ Below $40 \mathrm{~K}$, a strong discrepancy with this expression was observed and discussed in terms of a migration process of excitons. In this case, it was said that a thermalization process of the excitons could occur, which would lead to a preferential migration from small to big ncs. At low temperature, beyond $50 \mathrm{~K}$, this process becomes weaker and weaker and each nc contributes like an exciton trap. Then smaller crystals contribute to the PL signal, which could explain the strong increase in the PL energy at low temperature. A debate still exists as far as the migration of excitons in the $\mathrm{Si}$-ncs is concerned. In porous silicon, some authors have proposed that a migration process could happen between ncs, which could be at the origin of the stretched exponential decay of the PL intensity. ${ }^{22}$ However, some experimental and recent theoretical works strongly suggest that no carrier migration exists. ${ }^{23,24}$

In this present work, we demonstrate that the temperature dependence of the PL energy below $40 \mathrm{~K}$ can be interpreted without taking into account the contested migration process. Indeed, as shown above, the very long radiative lifetime at low temperature was induced in a saturation phenomenon. Moreover, the radiative lifetime is a decreasing function of the energy. As shown in Table I, the size distribution is narrow but still presents a dispersion, which probably contributes to the existence of a wide PL peak. The size distribution also involves a distribution of the radiative decay time because the quantum confinement energy is size dependent. Hence the saturation effect that appears at low temperature 
does not affect the small crystals as much as the large ones, as shown by the results obtained by the study of the temperature dependence of the PL intensity. At a given temperature, the large crystals feel a stronger saturation effect than the smaller ones, due to their larger lifetime. When the temperature decreases, the PL intensity of the large crystals decreases more rapidly than the PL intensity of the smaller ones. Consequently, when the saturation effect becomes strong, i.e., below around $40 \mathrm{~K}$, the PL signal comes essentially from the smaller ncs and the PL band shifts toward the higher energies, due to the preferential saturation effect. The strong increase at low temperature of the PL energy compared to the prediction of Pässler's law ${ }^{19}$ was obtained for all the studied samples, as demonstrated in Fig. 7. Moreover, the deviation with respect to this law is an increasing function of the size distribution. This energy increase is equal to 6 and $18 \mathrm{meV}$ for samples S2 and S5, respectively. This difference can be explained by the fact that, the larger the size dispersion is, the larger the dispersion on the lifetime is and the more pronounced the preferential saturation is. The shift at low temperature due to the saturation is consequently increased by the large size dispersion.

\section{CONCLUSIONS}

In summary, the temperature dependence of the PL properties of Si-ncs was studied for different Si-nc sizes. For this purpose, size-controlled Si-ncs were obtained using $\mathrm{SiO} / \mathrm{SiO}_{2}$ multilayers prepared by evaporation. The temperature dependences of the decay time, the PL intensity, and the PL energy were studied from 5 to $300 \mathrm{~K}$. The influence of the excitation power was also investigated. The PL intensity shows a maximum at a temperature, which is an increasing function of the power excitation. While the PL is strongly influenced by the nonradiative recombinations at temperatures higher than this maximum, the decrease in the PL intensity from 70 to $5 \mathrm{~K}$ is explained by a saturation effect, which is due to the strong increase in the PL radiative lifetime at low temperature. This saturation effect is more pronounced for the large Si-ncs because of their higher radiative lifetime.

The PL energy of the Si-ncs is a decreasing function of the temperature, which presents a stronger decrease from 5 to $40 \mathrm{~K}$. Taking into account the quantum confinement energy, the PL energy follows very well Varshni's law in the range 40-300 K. Below $40 \mathrm{~K}$, the strong discrepancy to this law was interpreted by a preferential saturation effect of the $\mathrm{Si}$ ncs that appends at low temperature. The small clusters present a smaller lifetime than the larger ones. Hence they do not feel the saturation effect as much as the larger ones, which induces a preferential decrease in the PL intensity in the low-energy part of the PL spectrum and therefore a shift of the spectrum toward the high energies.

${ }^{1}$ O. Bisi, S. Ossicini, and L. Pavesi, Surf. Sci. Rep. 38, 1 (2000).

${ }^{2}$ C. Delerue, G. Allan, and M. Lannoo, Phys. Rev. B 48, 11024 (1993).

${ }^{3}$ M. Zacharias, J. Heitmann, R. Scholz, U. Kahler, M. Schmidt, and J. Bläsing, Appl. Phys. Lett. 80, 661 (2002).

${ }^{4}$ C. Garcia, B. Garrido, P. Pellegrino, R. Ferre, J. A. Moreno, J. R. Morante, L. Pavesi, and M. Cazzanelli, Appl. Phys. Lett. 82, 1595 (2003).

${ }^{5}$ M. V. Wolkin, J. Jorne, P. M. Fauchet, G. Allan, and C. Delerue, Phys. Rev. Lett. 82, 197 (1999).

${ }^{6}$ X. X. Wang, J. G. Zhang, L. Ding, B. W. Cheng, W. K. Ge, J. Z. Yu, and Q. M. Wang, Phys. Rev. B 72, 195313 (2005).

${ }^{7}$ S. Godefroo, M. Hayne, M. Jivanescu, A. Stesmans, M. Zacharias, O. I. Lebedev, G. Van Tendeloo, and V. V. Moshchalkov, Nat. Nanotechnol. 3, 174 (2008)

${ }^{8}$ M. Sykora, L. Mangolini, R. D. Schaller, U. Kortshagen, D. Jurbergs, and V. I. Klimov, Phys. Rev. Lett. 100, 067401 (2008).

${ }^{9}$ P. D. J. Calcott, K. J. Nash, L. T. Canham, M. J. Kane, and D. Brumhead, J. Phys.: Condens. Matter 5, L91 (1993).

${ }^{10}$ A. G. Cullis, L. T. Canham, and P. D. J. Calcott, J. Appl. Phys. 82, 909 (1997).

${ }^{11}$ M. L. Brongersma, P. G. Kik, A. Polman, K. S. Min, and H. A. Atwater, Appl. Phys. Lett. 76, 351 (2000).

${ }^{12}$ Y. Kanemitsu, T. Ogawa, K. Shiraishi, and K. Takeda, Phys. Rev. B 48, 4883 (1993).

${ }^{13}$ V. Vinciguerra, G. Franzò, F. Priolo, F. Iacona, and C. Spinella, J. Appl. Phys. 87, 8165 (2000).

${ }^{14}$ J. Linnros, N. Lalic, A. Galeckas, and V. Grivickas, J. Appl. Phys. 86, 6128 (1999).

${ }^{15}$ T. Suemoto, K. Tanaka, and A. Nakajima, Phys. Rev. B 49, 11005 (1994).

${ }^{16}$ R. Rölver, M. Först, O. Winkler, B. Spangenberg, and H. Kurz, J. Vac. Sci. Technol. A 24, 141 (2006).

${ }^{17}$ D. Kovalev, H. Heckler, G. Polisski, and F. Koch, Phys. Status Solidi B 215, 871 (1999).

${ }^{18}$ Y. P. Varshni, Physica (Amsterdam) 34, 149 (1967).

${ }^{19}$ R. Pässler, J. Appl. Phys. 89, 6235 (2001).

${ }^{20}$ R. Pässler, Solid-State Electron. 39, 1311 (1996).

${ }^{21}$ J. Heitmann, F. Müller, L. Yi, M. Zacharias, D. Kovalev, and F. Eichhorn, Phys. Rev. B 69, 195309 (2004).

${ }^{22}$ L. Pavesi, J. Appl. Phys. 80, 216 (1996).

${ }^{23}$ I. Mihalcescu, J. C. Vial, and R. Romestain, Phys. Rev. Lett. 80, 3392 (1998).

${ }^{24}$ S. Tanaka, H. Koyama, and N. Koshida, Appl. Phys. Lett. 73, 2334 (1998).

${ }^{25}$ H. Rinnert, M. Vergnat, G. Marchal, and A. Burneau, Appl. Phys. Lett. 72, 3157 (1998).

${ }^{26}$ O. Jambois, H. Rinnert, X. Devaux, and M. Vergnat, J. Appl. Phys. 98, 046105 (2005).

${ }^{27}$ O. Jambois, H. Rinnert, X. Devaux, and M. Vergnat, J. Appl. Phys. 100, 123504 (2006).

${ }^{28}$ J. C. Vial, A. Bsiesy, F. Gaspard, R. Herino, M. Ligeon, F. Muller, R. Romestain, and R. M. MacFarlane, Phys. Rev. B 45, 14171 (1992).

${ }^{29}$ L. Pavesi and M. Ceschini, Phys. Rev. B 48, 17625 (1993).

${ }^{30}$ C. Delerue, G. Allan, C. Reynaud, O. Guillois, G. Ledoux, and F. Huisken, Phys. Rev. B 73, 235318 (2006).

${ }^{31}$ L. Viña, S. Logothetidis, and M. Cardona, Phys. Rev. B 30, 1979 (1984).

${ }^{32}$ F. Iacona, G. Franzo, and C. Spinella, J. Appl. Phys. 87, 1295 (2000). 